\title{
RESPONSABILIDAD SOCIAL CORPORATIVA EN LOS HOSPITALES CATALANES: ¿QUÉ NOS DICEN SUS WEBS?
}

\section{Corporate social responsibility in Catalan hospitals: what their websites tell us?}

Ana Rodríguez Cala ${ }^{1}$, Candela Calle Rodríguez, Barbara Zöller Grunembaun, Ana Pons Rodríguez*, Yolanda Benavente Moreno, Noemí Durán García

$$
\begin{aligned}
& \text { Institut Català d'Oncologia (ICO) } \\
& \text { *Institut Català de la Salut (ICS) }
\end{aligned}
$$

\section{Resumen}

La Responsabilidad Social Corporativa (RSC), es una estrategia de gestión empresarial que adquiere cada vez más importancia en los centros hospitalarios. Los hospitales, al igual que otras organizaciones, desarrollan iniciativas de RSC y hacen difusión de las mimas a través de la Web corporativa. El objetivo de este artículo es analizar la información de RSC de los hospitales públicos catalanes, mediante la web corporativa, para conocer el compromiso que tiene la organización con sus grupos de interés. Método. Se analizaron 60 webs de hospitales de la red pública hospitalaria de Cataluña. Resultados. Todos los hospitales publican en sus webs criterios de RSC, aunque con cierta divergencia. Los aspectos más comunes fueron el sistema de gestión de calidad, la publicación de las cuentas y el código ético. La aplicación y difusión de la RSC en los hospitales de la red pública catalana es desigual, todos implementan algún criterio pero todavía queda mucho por hacer.

Palabras Claves: benchmarking; hospital; responsabilidad social corporativa; RSC; salud 2.0; web

\begin{abstract}
Corporate Social Responsibility (CSR) is a business management strategy that is becoming increasingly important in the hospitals. Hospitals, like other organizations, develop initiatives of CSR and make dissemination through the corporate website. The aim of this paper is to analyze the information of CSR provided by the Catalan public hospitals through their corporate website, in order to know the commitment of the organization with its stakeholders.

Methodology. Analysis of 60 websites of hospitals of the public hospital network in Catalonia. Results. All hospitals publish on their websites CSR criteria, although with some divergence. The most common issues are the system of quality management, the publication of the accounts and the ethical code. The
\end{abstract}

\footnotetext{
1Autor de contacto: Ana Rodríguez Cala arcala@iconcologia.net
} 
Responsabilidad social corporativa en los hospitales catalanes: ¿qué nos dicen sus webs?

implementation and dissemination of the RSC in the hospitals of the Catalan public network is uneven. They all implemented some criterion, but much remains to be done.

Keywords: benchmarking; hospital; corporate social responsibility; CSR; health 2.0; Web

\section{Como citar el artículo}

Rodríguez Cala, A.; Calle Rodríguez, C.; Zöller Grunembaun, B.; Pons Rodríguez, A.; Benavente Moreno, Y.; Durán García, N. (2017). Responsabilidad social corporativa en los hospitales catalanes: ¿qué nos dicen sus webs?. Revista de Comunicación y Salud, vol. 7, pp. 13-28. Recuperado de http://revistadecomunicacionysalud.org/index.php/rcys/article/view/106

\section{INTRODUCCIÓN}

La Responsabilidad Social Corporativa (RSC) se puede definir como el comportamiento que debe adoptar una empresa ante sus grupos de interés y la sociedad en su conjunto, lo que obliga a cumplir determinados compromisos (Marin Calahorro, 2008:9). Desde una perspectiva ética y filosofía empresarial, la RSC responde a la pregunta qué sociedad queremos construir y cuál es el papel de los actores que forman parte de ella. En otras palabras, la RSC hace referencia a todas aquellas acciones que desarrolla una empresa que desea ser percibido como una organización responsable.

La RSC es una forma de dirigir una empresa, en la que ella y las partes interesadas, marcan unos objetivos a cumplir (a corto, medio o largo plazo), -un enfoque estratégico- teniendo en cuenta las tres dimensiones Triple Botton Line $^{1}$ (la económica, la social y la medioambiental). $Y$ todo ello utilizando una comunicación con la máxima transparencia.

Cada vez aumenta más el número de empresas que adoptan criterios de RSC y que utilizan la web corporativa para difundir las buenas prácticas y los informes de sostenibilidad. Prueba de ello es que entre 1999 y 2012 el número de empresas suscritas al Pacto Mundial de Naciones Unidas (www.unglobalcompact.org) se ha incrementado de 38 a12.099 entidades/empresas de 140 países, de las cuales 1.701 son españolas. (Annual Local Network Report 2013 y el Corporate Sustainibility Report 2013)

Los centros sanitarios, inicialmente, han implementado acciones de RSC con el objetivo de mejorar la salud, dar una asistencia de calidad y garantizar la seguridad de los pacientes. Sin embargo no todos los centros conciben la RSC como un concepto global, que envuelve a toda la organización y que incluye diferentes acciones de índole económica, social y ambiental.

Todavía para la mayoría de los centros, supone el reto de abordar la gestión teniendo en cuenta dos perspectivas: una holística, partiendo de la propia práctica asistencial como elemento central de las actividades hospitalarias y otra estratégica que implica la gestión de los recursos humanos, la política de compras, la logística, el consumo de energía, la gestión de residuos y la movilidad de los pacientes y familiares.

Los centros hospitalarios catalanes, están organizados por el Servei Català de la Salut (Departament de Salut de la Generalitat de Catalunya) en la llamada 
Red Hospitalaria de Utilización Pública (XHUP, por sus siglas en catalán), y entre ellos encontramos fundaciones privadas, empresas públicas, consorcios, etc. La gestión puede ser pública, privada o mixta. Principalmente el servicio sanitario que se ofrece es solo público y en el caso de ser privado, se puede concertar con el CatSalut (servicio público) o bien realizado a través de mutuas (totalmente privado).

Las nuevas tecnologías han favorecido la comunicación uni y bi-direccional entre los centros sanitarios y la sociedad y sus usuarios. Es importante recalcar, que los hospitales tienen una clara orientación hacia los pacientes/ usuarios y que sus webs son una herramienta fundamental para divulgar sus políticas de RSC, su misión, sus códigos y sus resultados. La web es una herramienta de comunicación que facilita el llegar no solo a todos los grupos de interés sino a toda la ciudadanía.

El objetivo de nuestro estudio es identificar las buenas prácticas de RSC publicadas en la web corporativa de los hospitales de la Red Hospitalaria de Utilización Pública (XHUP), y hacer benchmarking entre centros para identificar las mejores prácticas señalando los patrones de referencia.

\section{METODOLOGÍA}

Se realizó un estudio observacional transversal de las prácticas de RSC publicadas en las webs corporativas de 60 hospitales de la XHUP. Se incluyeron todos los hospitales con web corporativa entre junio-septiembre de 2013. Se diseñó un formulario para la recogida de datos que incluía información general sobre los hospitales (descripción, dirección web y la región), e información específica sobre la RSC. Se recogieron datos sobre 25 ítems de RSC que se agruparon en cinco apartados: 1) aspectos generales de RSC (política de RSC, plan estratégico, sistema de gestión de calidad, memoria de sostenibilidad y adhesión al Pacto Mundial); 2) aspectos económicos (código de buen gobierno y rendición de cuentas); 3) aspectos sociales conciliación de la vida familiar y laboral, plan de igualdad, código ético, gestión de la diversidad, proyectos solidarios, voluntariado corporativo); 4) aspectos medioambientales (política medioambiental y gestión medioambiental); y 5) certificaciones (GRI, SA 8000, ISO 14001, EMAS, EFQM, ISO 26.000, AA 1000, SG21). La selección de los ítems se realizó a partir de los criterios de Global Reporting Initiative (www.globalreporting.org) y Pacto Mundial de Naciones Unidas (www.unglobalcompact.org).

La revisión de las webs corporativa fue realizada por dos investigadoras, tras un entrenamiento previo de revisión conjunta de las distintas webs y establecimiento de criterios comunes para minimizar la variabilidad en la recogida de la información. El criterio de inclusión fue que los hospitales tuviesen página web propia. De 69 hospitales seleccionados, finalmente se han incluido 60 en el análisis porque algunos de ellos pertenecían a un consorcio y disponían de web única.

Para cada centro se ha calculado el número de ítems sobre un máximo de 25. Se ha descrito este número de ítems de forma global y por apartado para cada uno de los centros así como por región sanitaria. La descripción se ha realizado de forma gráfica (diagrama de cajas) y por medio de estadísticos descriptivos de acuerdo con la naturaleza de la variable (número de 
observaciones no-missing, la media, la desviación estándar, el primer cuartil, la mediana, el tercer cuartil y el máximo). Para evaluar la variabilidad entre centros se ha estimado la razón de variación en el percentil 20 y 80 .El análisis estadístico se realizó con SPSS.19.0

\section{RESULTADOS}

Los 60 hospitales analizados representan el $86,96 \%$ de los hospitales de XHUP y todos difunden en mayor o menor grado actividades de RSC en sus webs corporativas. Por regiones sanitarias el $50 \%$ de los hospitales fue de Barcelona, el $15 \%$ de Girona, el $10 \%$ de Camp de Tarragona y el $25 \%$ restante de las regiones sanitarias de Alt Pirineu i Aran, Catalunya Central, Lleida y Terres de l'Ebre.

En la figura 1 se muestra la distribución de los hospitales por región sanitaria.

Figura 1. Distribución de la muestra de hospitales por región sanitaria

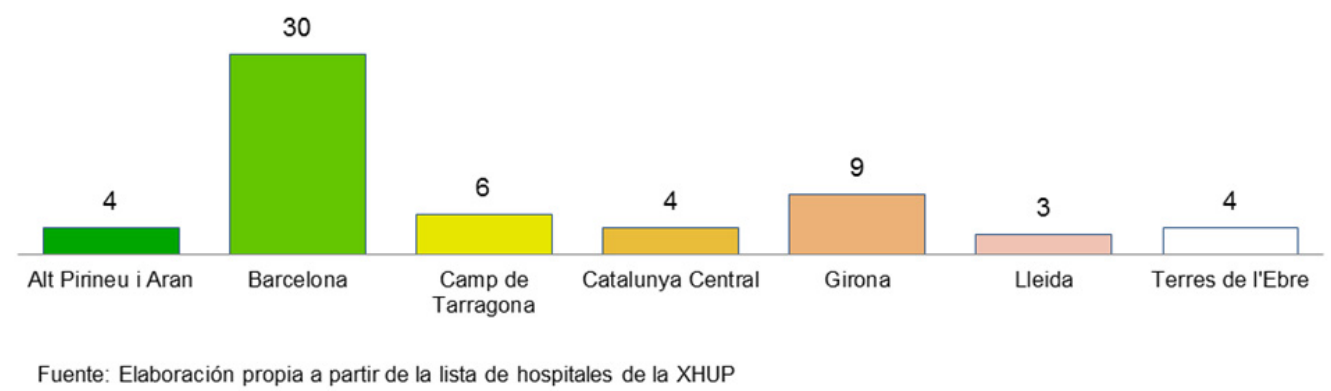

En la figura 2 se presenta el diagrama de cajas y la función de densidad del número de ítems identificados en la web de cada centro (cada punto representa un centro). De los 60 centros analizados el promedio de aspectos identificados es de 5,9 (desviación estándar 4,4 ) sobre un máximo de 25 . El $50 \%$ de los centros presentaba al menos 4 aspectos, había un $25 \%$ con 9 o más y otro $25 \%$ con menos de 3 aspectos. La razón de variación (RV20-80 =5,5) entre los centros sugiere una importante variabilidad cuantificable en más 5 veces más ítems identificados en los centros en el percentil 80 respecto el percentil 20.

Figura 2. Número de ítems identificados por centro
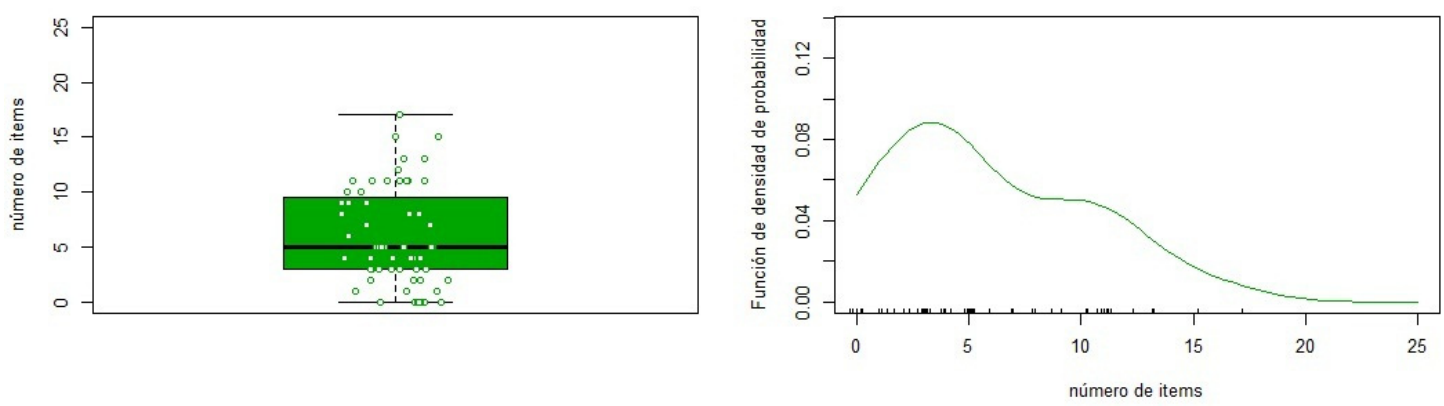

Fuente: Elaboración propia a partir de datos extraídos de las webs corporativas de los hospitales 
Responsabilidad social corporativa en los hospitales catalanes: ¿qué nos dicen sus webs?

En la figura 3 se muestra el diagrama de cajas con los resultados estratificados por región sanitaria, donde se puede observar cierta homogeneidad en los aspectos observados en las web de los hospitales por regiones, excepto Camp de Tarragona, donde se presenta una mayor variabilidad.

Figura 3. Número de ítems identificados por región sanitaria

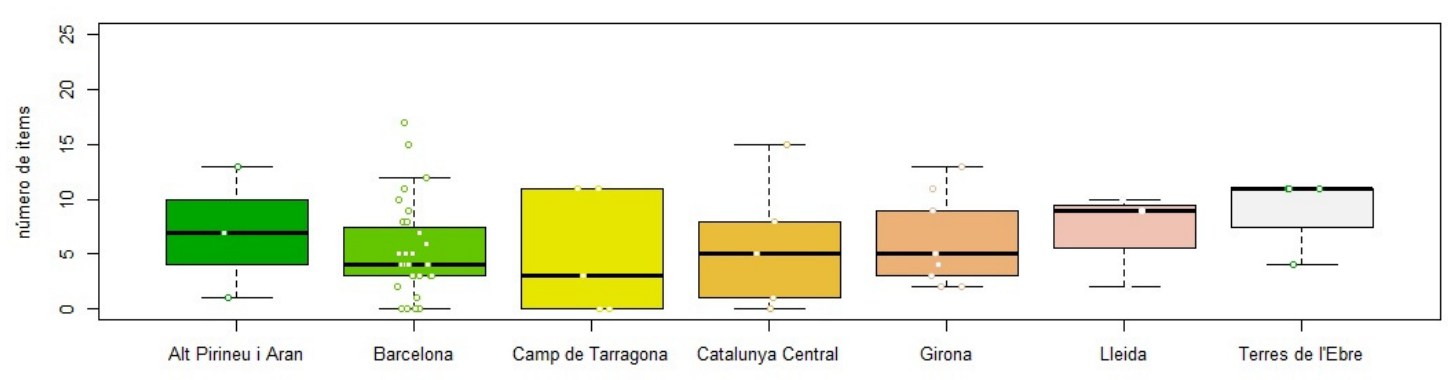

Fuente: Elaboración propia a partir de datos extraídos de las webs corporativas de los hospitales

Todos los hospitales analizados difunden en mayor o menor grado actividades de RSC en sus webs corporativas. Aunque existe cierta variabilidad en los contenidos.

En el primer lugar nos hemos centrado en los Aspectos generales

En relación con los aspectos generales observados, el 43,33\% $(n=26)$ de los hospitales cuentan con un plan estratégico, público a través de su web corporativa, el $26,67 \%(n=16)$ disponen de una política de RSC y el $5,00 \%$ $(n=3)$ tienen dirección o departamento de RSC.

En cuanto a otros aspectos, el $58,33 \%(n=34)$ de los hospitales observados disponen de un sistema de gestión de calidad, entre los cuales destacan los de las regiones de Terres de l'Ebre, Girona y Lleida, como se puede observar en la figura 4, donde se muestra el porcentaje de implementación de aspectos generales en los hospitales por región sanitaria. Todos los hospitales observados publican sus memorias anuales de sostenibilidad, pero el porcentaje que realiza memorias de sostenibilidad de acuerdo a los criterios del Global Reporting Initiative (GRI) es del $28,33 \%(n=17)$. Y solo, el $18,33 \%$ $(n=11)$ de los hospitales están adheridos al Pacto Mundial ${ }^{2}$.

Figura 4. Implementación de Aspectos generales por región sanitaria

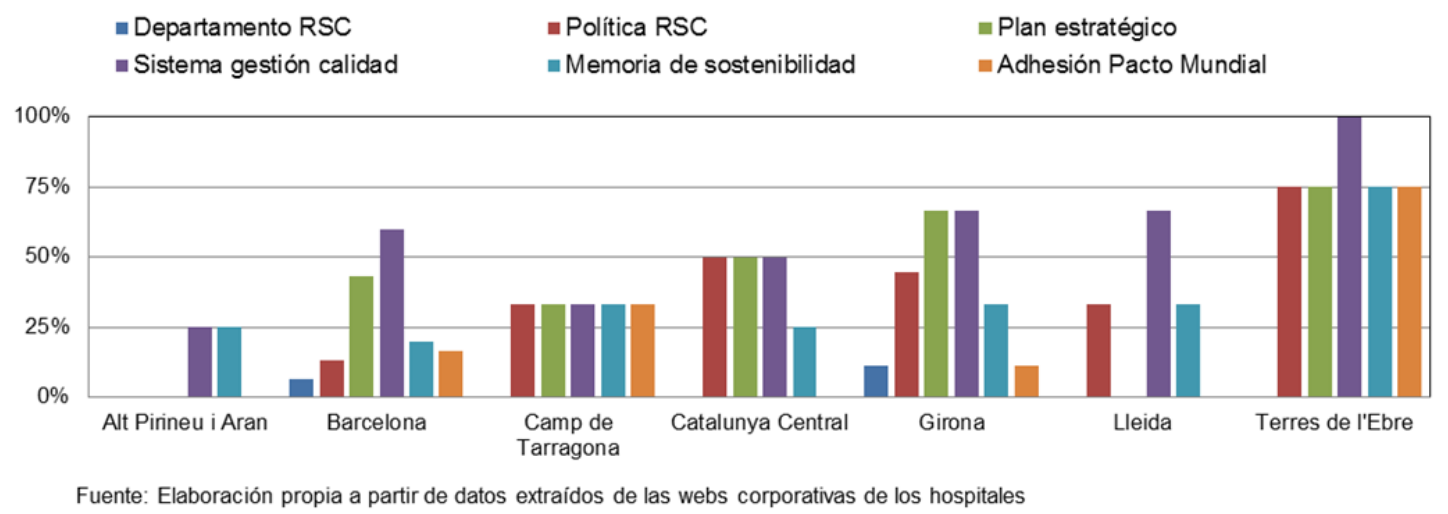


Responsabilidad social corporativa en los hospitales catalanes: ¿qué nos dicen sus webs?

En segundo lugar damos información sobre los Aspectos económicos

Los aspectos económicos muestran un patrón desigual. El 8,33\% $(n=5)$ de los centros analizados tienen publicado en su web el código de buen gobierno. Sin embargo, más de la mitad $(n=30 ; 55,33 \%)$ de los hospitales de la red pública rinden cuentas en la web corporativa. No obstante, existen distintos grado de implantación, como se aprecia en la figura 5.

Figura 5. Implementación de Aspectos económicos por región sanitaria

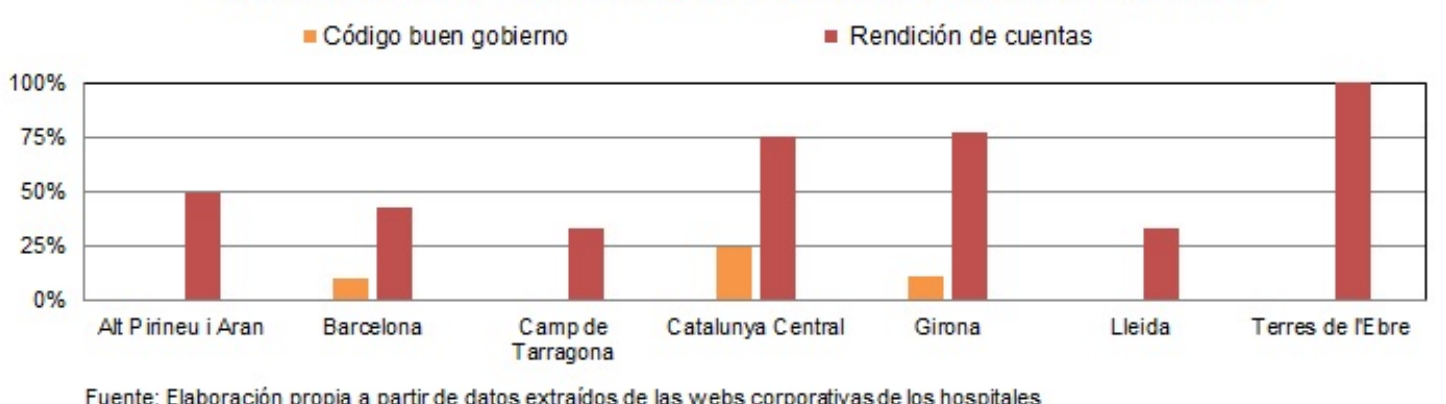

En tercer lugar contemplamos los Aspectos sociales

Dentro de los aspectos sociales, los observados con más frecuencia en las webs de los hospitales de la XHUP son el código ético $(51,67 \% ; n=31)$ y la gestión de la diversidad $(41,67 \% ; n=25)$. Les siguen las acciones para conciliar la vida laboral y familiar $(26,67 \%$; $n=16)$, los proyectos solidarios $(26,67 \%$; $\mathrm{n}=16)$ y el plan de igualdad $(18,33 \% ; \mathrm{n}=11)$. Por último, el programa de voluntariado se encuentra presente en menos del $5 \%$ de los centros de la XHUP $(n=2 ; 3,33 \%)$. En referencia a la implementación por región sanitaria, como se observa en la figura 6 , es desigual.

Figura 6. Implementación de Aspectos sociales por región sanitaria

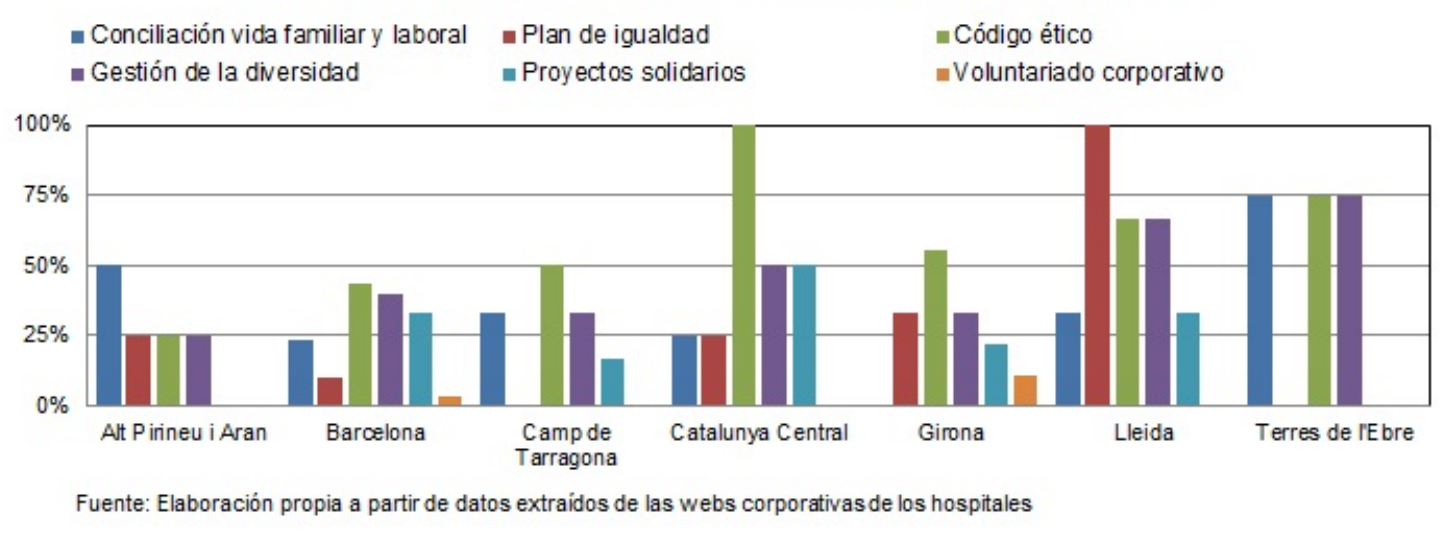

En cuarto lugar damos información sobre los Aspectos medioambientales

Se observó también que el $35,00 \%(n=21)$ de los hospitales tienen política medioambiental donde destacan los hospitales de Terres de l'Ebre y Catalunya Central, como se refleja en la figura 7 donde se muestra el porcentaje de implementación de aspectos medioambientales en los hospitales por región sanitaria. 
Responsabilidad social corporativa en los hospitales catalanes: ¿qué nos dicen sus webs?

El 43,33\% $(n=26)$ de los hospitales disponen de un sistema de gestión medioambiental. En este caso, además de los hospitales de Terres de l'Ebre y Catalunya Central, destacan los centros de Lleida y Camp de Tarragona.

Figura 7. Implementación de Aspectos medioambientales por región sanitaria

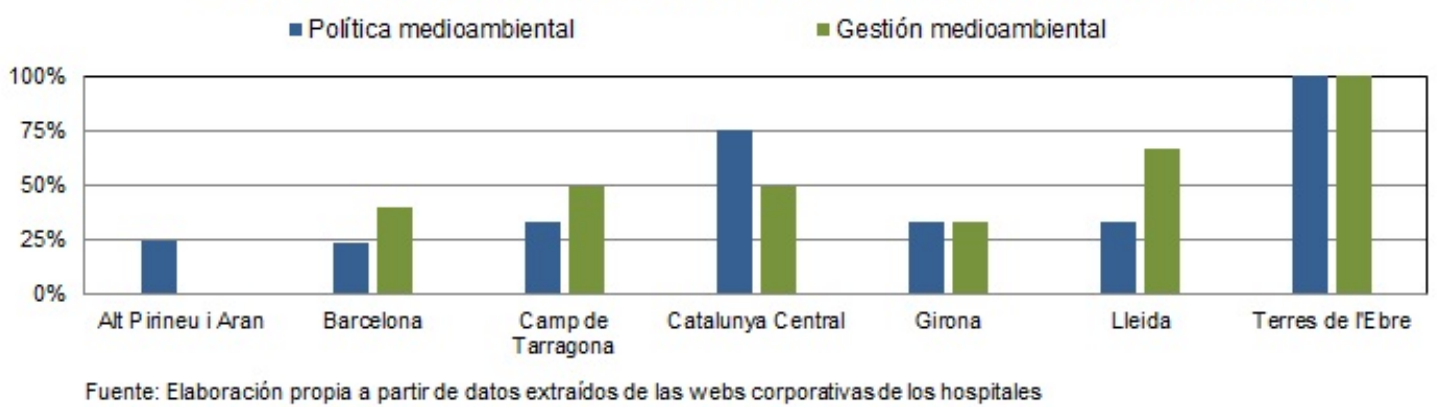

El quinto apartado analiza las Certificaciones que tiene el centro hospitalario

Las certificaciones más utilizadas en los centros sanitarios son GRI $(15,00 \%$; $n=9), \operatorname{EMAS}^{3}(3,33 \% ; n=2)$, ISO $14001(5,00 \% ; n=3)$ y $\operatorname{EFQM}^{4}(5,00 \% ; n=3)$, entre los cuales cabe destacar el porcentaje de Memoria GRI. GRI es el principal referente para la publicación de información de sostenibilidad, cuya guía tiene como objetivo satisfacer la necesidad de comunicar de forma transparente sobre la sostenibilidad de las empresas con independencia de su tamaño, sector o ubicación.

La tabla 1 recoge todos y cada uno de los aspectos analizados de las webs corporativas de los hospitales estudiados.

\section{Tabla 1}

Información de RSC en las webs de los hospitales públicos de Cataluña, 2013

\begin{tabular}{lcc}
\hline \multicolumn{1}{c}{ Ítems } & $\mathrm{n}$ & $\%$ \\
\hline Aspectos generales & 3 & $5,00 \%$ \\
\hline 1. Tiene director o departamento de RSC & 16 & $26,67 \%$ \\
\hline 2. Tiene política de RSC & 26 & $43,33 \%$ \\
\hline 3. Tiene plan estratégico & 34 & $58,33 \%$ \\
\hline 4. Dispone de un sistema de gestión de calidad & 17 & $28,33 \%$ \\
\hline 5. Realiza memoria de sostenibilidad & 11 & $18,33 \%$ \\
\hline 6. Está adherido al Pacto Mundial & & \\
\hline Aspectos económicos & 5 & $8,33 \%$ \\
\hline 7. Dispone de código de buen gobierno & 30 & $53,33 \%$ \\
\hline 8. Presenta su rendimiento de cuentas & 31 & \\
\hline Aspectos sociales & $51,67 \%$ \\
\hline 9. Dispone de código ético & 11 & $18,33 \%$ \\
\hline 10. Tiene plan de lgualdad & 16 & $26,67 \%$ \\
\hline 11. Se preocupa de la conciliación vida laboral y familiar & 25 & $41,67 \%$ \\
\hline 12. Gestiona la diversidad & 16 & $26,67 \%$ \\
\hline 13. Tiene proyectos solidarios & 2 & $3,33 \%$ \\
\hline 14. Realiza programa de voluntariado corporativo & & \\
\hline
\end{tabular}


Responsabilidad social corporativa en los hospitales catalanes: ¿qué nos dicen sus webs?

\begin{tabular}{|c|c|c|}
\hline \multicolumn{3}{|l|}{ Aspectos medioambientales } \\
\hline 15. Dispone de política medio ambiental & 21 & $35,00 \%$ \\
\hline 16. Realiza acciones para gestionar el medio ambiental & 26 & $43,33 \%$ \\
\hline \multicolumn{3}{|l|}{ Certificaciones y/o cumplimento de estándares } \\
\hline $\begin{array}{l}\text { 17. Verificación externa memoria de sostenibilidad de acuerdo a } \\
\text { GRI }\end{array}$ & 8 & $15,00 \%$ \\
\hline 18. Modelo de excelencia y calidad EFQM & 3 & $5,00 \%$ \\
\hline 19. Sistema de gestión ambiental ISO 14001 & 3 & $5,00 \%$ \\
\hline 20. Sistema de gestión ambiental EMAS & 7 & $3,33 \%$ \\
\hline 21. Sistema de gestión ética y socialmente responsable SGE21 & 0 & $0,00 \%$ \\
\hline 22. Principios de AcountabilityAA1000 & 0 & $0,00 \%$ \\
\hline 23. Principios de responsabilidad social ISO 26000 & 0 & $0,00 \%$ \\
\hline $\begin{array}{l}\text { 24. Sistema de gestión de seguridad y salud en el trabajo OHSAS } \\
18001\end{array}$ & 0 & $0,00 \%$ \\
\hline 25. Código de conducta SA 8000 & 0 & $0,00 \%$ \\
\hline
\end{tabular}

Fuente: Elaboración propia a partir de datos extraídos de las webs corporativas de los hospitales

Con los resultados obtenidos hemos querido destacar los 10 hospitales que presentaron los mejores patrones de referencia a tener en cuenta para desarrollar una guía de mínimos de comunicación de la RSC para los hospitales catalanes (Tabla 2). Se han seleccionado los hospitales que disponen de más de un $50 \%$ de los ítems explorados en los aspectos generales, económicos, sociales y medioambientales (Tabla 3).

Tabla 2

Hospitales catalanes con mejores patrones de referencia de RSC, 2013

\begin{tabular}{|c|c|c|c|c|c|}
\hline & $\begin{array}{l}\text { Aspectos } \\
\text { generales }\end{array}$ & $\begin{array}{l}\text { Aspectos } \\
\text { económico } \\
s\end{array}$ & $\begin{array}{l}\text { Aspectos } \\
\text { sociales }\end{array}$ & $\begin{array}{l}\text { Aspectos } \\
\text { medioambient } \\
\text { ales }\end{array}$ & $\begin{array}{l}\text { Certificacio } \\
\text { nes }\end{array}$ \\
\hline 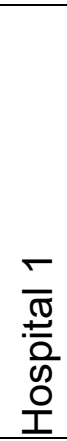 & $\begin{array}{l}\text { Departamento } \\
\text { RSC } \\
\text { Plan estratégico } \\
\text { Sistema gestión } \\
\text { calidad } \\
\text { Memoria de } \\
\text { sostenibilidad } \\
\text { Adhesión Pacto } \\
\text { Mundial }\end{array}$ & $\begin{array}{l}\text { Rendición } \\
\text { de cuentas }\end{array}$ & $\begin{array}{l}\text { Código ético } \\
\text { Conciliación } \\
\text { vida familiar y } \\
\text { laboral } \\
\text { Plan igualdad } \\
\text { Gestión } \\
\text { diversidad } \\
\text { Proyectos } \\
\text { solidarios } \\
\end{array}$ & $\begin{array}{l}\text { Política medio } \\
\text { ambiental } \\
\text { Gestión } \\
\text { medioambient } \\
\text { al }\end{array}$ & $\begin{array}{l}\text { GRI } \\
\text { EFQM }\end{array}$ \\
\hline$N$ & $\begin{array}{l}\text { Política de RSC } \\
\text { Plan estratégico } \\
\text { Sistema gestión } \\
\text { calidad } \\
\text { Memoria de } \\
\text { sostenibilidad }\end{array}$ & $\begin{array}{l}\text { Código de } \\
\text { buen } \\
\text { gobierno } \\
\text { Rendición } \\
\text { de cuentas }\end{array}$ & $\begin{array}{l}\text { Código ético } \\
\text { Conciliación } \\
\text { vida familiar y } \\
\text { laboral } \\
\text { Gestión } \\
\text { diversidad } \\
\text { Proyectos } \\
\text { solidarios }\end{array}$ & $\begin{array}{l}\text { Política medio } \\
\text { ambiental } \\
\text { Gestión } \\
\text { medioambient } \\
\text { al }\end{array}$ & $\begin{array}{l}\text { GRI } \\
\text { ISO } 14001 \\
\text { EMAS }\end{array}$ \\
\hline
\end{tabular}




\begin{tabular}{|c|c|c|c|c|c|}
\hline $\begin{array}{l}\frac{m}{\pi} \\
\frac{\pi}{\overline{0}} \\
\frac{0}{10} \\
\text { 은 }\end{array}$ & $\begin{array}{l}\text { Departamento } \\
\text { RSC } \\
\text { Política de RSC } \\
\text { Plan estratégico } \\
\text { Sistema gestión } \\
\text { calidad } \\
\text { Memoria de } \\
\text { sostenibilidad }\end{array}$ & $\begin{array}{l}\text { Código de } \\
\text { buen } \\
\text { gobierno } \\
\text { Rendición } \\
\text { de cuentas }\end{array}$ & $\begin{array}{l}\text { Código ético } \\
\text { Conciliación } \\
\text { vida familiar y } \\
\text { laboral } \\
\text { Plan igualdad } \\
\text { Gestión } \\
\text { diversidad } \\
\text { Proyectos } \\
\text { solidarios }\end{array}$ & $\begin{array}{l}\text { Política medio } \\
\text { ambiental } \\
\text { Gestión } \\
\text { medioambient } \\
\text { al }\end{array}$ & \\
\hline 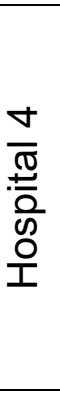 & $\begin{array}{l}\text { Política de RSC } \\
\text { Plan estratégico } \\
\text { Sistema gestión } \\
\text { calidad } \\
\text { Memoria de } \\
\text { sostenibilidad } \\
\text { Adhesión Pacto } \\
\text { Mundial }\end{array}$ & $\begin{array}{l}\text { Código de } \\
\text { buen } \\
\text { gobierno } \\
\text { Rendición } \\
\text { de cuentas }\end{array}$ & $\begin{array}{l}\text { Código ético } \\
\text { Plan igualdad } \\
\text { Gestión } \\
\text { diversidad } \\
\text { Proyectos } \\
\text { solidarios }\end{array}$ & $\begin{array}{l}\text { Política medio } \\
\text { ambiental } \\
\text { Gestión } \\
\text { medioambient } \\
\text { al }\end{array}$ & \\
\hline $\begin{array}{l}\frac{10}{\pi} \\
\frac{0}{\overline{0}} \\
\frac{0}{10} \\
\frac{0}{1}\end{array}$ & $\begin{array}{l}\text { Plan estratégico } \\
\text { Sistema gestión } \\
\text { calidad } \\
\text { Memoria de } \\
\text { sostenibilidad }\end{array}$ & $\begin{array}{l}\text { Rendición } \\
\text { de cuentas }\end{array}$ & $\begin{array}{l}\text { Código ético } \\
\text { Conciliación } \\
\text { vida familiar y } \\
\text { laboral } \\
\text { Plan igualdad } \\
\text { Gestión } \\
\text { diversidad }\end{array}$ & $\begin{array}{l}\text { Política medio } \\
\text { ambiental } \\
\text { Gestión } \\
\text { medioambient } \\
\text { al }\end{array}$ & $\begin{array}{l}\text { GRI } \\
\text { ISO } 14001\end{array}$ \\
\hline $\begin{array}{l}\frac{0}{\sigma} \\
\frac{\pi}{\overline{0}} \\
\text { की } \\
\text { 운 }\end{array}$ & $\begin{array}{l}\text { Política de RSC } \\
\text { Plan estratégico } \\
\text { Sistema gestión } \\
\text { calidad } \\
\text { Memoria de } \\
\text { sostenibilidad } \\
\text { Adhesión al Pacto } \\
\text { Mundial }\end{array}$ & $\begin{array}{l}\text { Rendición } \\
\text { de cuentas }\end{array}$ & $\begin{array}{l}\text { Código ético } \\
\text { Conciliación } \\
\text { vida familiar y } \\
\text { laboral } \\
\text { Gestión } \\
\text { diversidad }\end{array}$ & $\begin{array}{l}\text { Política medio } \\
\text { ambiental } \\
\text { Gestión } \\
\text { medioambient } \\
\text { al }\end{array}$ & GRI \\
\hline $\begin{array}{l}\frac{1}{\pi} \\
\frac{\pi}{\overline{0}} \\
\frac{0}{0} \\
\frac{0}{1}\end{array}$ & $\begin{array}{l}\text { Política de RSC } \\
\text { Plan estratégico } \\
\text { Sistema gestión } \\
\text { calidad } \\
\text { Memoria de } \\
\text { sostenibilidad } \\
\text { Adhesión Pacto } \\
\text { Mundial }\end{array}$ & $\begin{array}{l}\text { Rendición } \\
\text { de cuentas }\end{array}$ & $\begin{array}{l}\text { Código ético } \\
\text { Conciliación } \\
\text { vida familiar y } \\
\text { laboral } \\
\text { Gestión } \\
\text { diversidad }\end{array}$ & $\begin{array}{l}\text { Política medio } \\
\text { ambiental } \\
\text { Gestión } \\
\text { medioambient } \\
\text { al }\end{array}$ & GRI \\
\hline $\begin{array}{l}\frac{\infty}{\frac{\pi}{\pi}} \\
\frac{0}{\frac{0}{0}} \\
\frac{0}{1} \\
\frac{1}{1}\end{array}$ & $\begin{array}{l}\text { Plan estratégico } \\
\text { Sistema gestión } \\
\text { calidad } \\
\text { Memoria de } \\
\text { sostenibilidad }\end{array}$ & $\begin{array}{l}\text { Rendición } \\
\text { de cuentas }\end{array}$ & $\begin{array}{l}\text { Código ético } \\
\text { Gestión } \\
\text { diversidad } \\
\text { Proyectos } \\
\text { solidarios }\end{array}$ & $\begin{array}{l}\text { Política medio } \\
\text { ambiental } \\
\text { Gestión } \\
\text { medioambient } \\
\text { al }\end{array}$ & $\begin{array}{l}\text { ISO } 14001 \\
\text { EMAS }\end{array}$ \\
\hline $\begin{array}{l}\frac{\sigma}{\bar{\sigma}} \\
\frac{\pi}{\overline{0}} \\
\text { की } \\
\text { 운 }\end{array}$ & $\begin{array}{l}\text { Política de RSC } \\
\text { Sistema gestión } \\
\text { calidad } \\
\text { Memoria de }\end{array}$ & $\begin{array}{l}\text { Rendición } \\
\text { de cuentas }\end{array}$ & $\begin{array}{l}\text { Código ético } \\
\text { Conciliación } \\
\text { vida familiar y } \\
\text { laboral }\end{array}$ & $\begin{array}{l}\text { Política medio } \\
\text { ambiental } \\
\text { Gestión } \\
\text { medioambient }\end{array}$ & \\
\hline
\end{tabular}


Responsabilidad social corporativa en los hospitales catalanes: ¿qué nos dicen sus webs?

sostenibilidad

Plan igualdad al

Gestión

diversidad

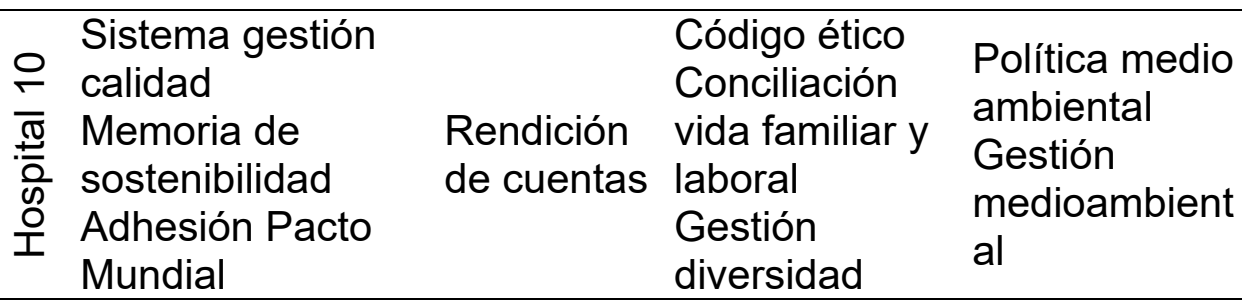

Fuente: Elaboración propia a partir de datos extraídos de las webs corporativas de los hospitales

Tabla 3

Puntuación de los hospitales con mejores patrones de referencia de RSC, 2013

\begin{tabular}{ccccccc}
\hline & $\begin{array}{c}\text { Puntuación } \\
\text { total }\end{array}$ & $\begin{array}{c}\text { Aspectos } \\
\text { generales }\end{array}$ & $\begin{array}{c}\text { Aspectos } \\
\text { económicos }\end{array}$ & $\begin{array}{c}\text { Aspectos } \\
\text { sociales }\end{array}$ & $\begin{array}{c}\text { Aspectos } \\
\text { medioambientales }\end{array}$ & $\begin{array}{c}\text { Certificacio- } \\
\text { nes }\end{array}$ \\
\hline $\begin{array}{c}\text { Hospital } \\
\text { Hospital }\end{array}$ & $15 / 25$ & $5 / 6$ & $1 / 2$ & $5 / 6$ & $2 / 2$ & $2 / 9$ \\
\hline $\begin{array}{c}\text { Hospital } \\
3\end{array}$ & $15 / 25$ & $4 / 6$ & $2 / 2$ & $4 / 6$ & $2 / 2$ & $3 / 9$ \\
\hline $\begin{array}{c}\text { Hospital } \\
4\end{array}$ & $13 / 25$ & $5 / 6$ & $2 / 2$ & $4 / 6$ & $2 / 2$ & $0 / 9$ \\
\hline $\begin{array}{c}\text { Hospital } \\
5\end{array}$ & $12 / 25$ & $3 / 6$ & $1 / 2$ & $4 / 6$ & $2 / 2$ & $2 / 9$ \\
\hline $\begin{array}{c}\text { Hospital } \\
6\end{array}$ & $12 / 25$ & $5 / 6$ & $1 / 2$ & $3 / 6$ & $2 / 2$ & $1 / 9$ \\
\hline $\begin{array}{c}\text { Hospital } \\
7\end{array}$ & $12 / 25$ & $5 / 6$ & $1 / 2$ & $3 / 6$ & $2 / 2$ & $1 / 9$ \\
\hline $\begin{array}{c}\text { Hospital } \\
8\end{array}$ & $11 / 25$ & $3 / 6$ & $1 / 2$ & $3 / 6$ & $2 / 2$ & $2 / 9$ \\
\hline $\begin{array}{c}\text { Hospital } \\
9\end{array}$ & $10 / 25$ & $3 / 6$ & $1 / 2$ & $4 / 6$ & $2 / 2$ & $0 / 9$ \\
\hline $\begin{array}{c}\text { Hospital } \\
10\end{array}$ & $9 / 25$ & $3 / 6$ & $1 / 2$ & $3 / 6$ & $2 / 2$ & $0 / 9$ \\
\hline
\end{tabular}

Fuente: Elaboración propia a partir de datos extraídos de las webs corporativas de los hospitales

\section{DISCUSIÓN}

En este estudio se evalúa la disponibilidad de información sobre RSC en las web corporativas de 60 hospitales de la XHUP en el período junio-septiembre 2013. De los resultados y el análisis de nuestro estudio se desprende que la web se ha convertido en un instrumento imprescindible para informar y comunicar actividades de RSC. Todos los hospitales incluidos hacen difusión de sus acciones de RSC en su web corporativa, sin embargo existe cierta variabilidad en la cantidad de información y en las acciones que realizan. 
Queremos destacar que en algunas webs fue necesario navegar a través de diferentes secciones de la web para poder tener una visión completa y global de todos las acciones de RSC e incluso para profundizar en algunos aspectos.

Los resultados indican que la web corporativa de los hospitales catalanes asume una función importante en información, pero no ofrecen la posibilidad de interaccionar con los grupos de interés. Podemos destacar, que los hospitales se encuentran en una fase inicial de información de RSC y que la utilización de la web en la actualidad es parcial, centrándose básicamente en dar información de código de buenas prácticas, de plan de igualdad, de la memoria de sostenibilidad, de la política de RSC y de las actividades sociales que realizan.

Algunos autores, ya desde los años 80 estudiaron la forma de cómo comunicar efectivamente la RSC (Bruning y Lendinghan, 1999; Dawkins, 2004; Grunig, 1979; Ingehoff, 2004; Manhein y Pratt, 1986), pero hay pocas investigaciones que hayan tratado de la importancia de las webs corporativas para comunicar sus acciones. Según Alison Clark (2000), internet permite una comunicación estratégica con los grupos de interés. Esrock \& Leichty (1998) probaron que el $82 \%$ de corporaciones del índice Fortune en US utilizaban la web para informar sobre RSC. En su trabajo se pone de manifiesto la importancia de la web como medio por sus características de velocidad de diseminación, acceso y retroalimentación. Estos autores plantearon que las webs daban una información valiosa sobre RSC. Otras investigaciones de UNEP (Corporate Environment Report, 2000) describen que hay compañías que tienen sus informes de RSC medioambiental anexados a sus webs. Maignan \& Ralston (2002) analizaron y compararon la comunicación sobre RSC en páginas webs corporativas de Francia, Holanda, Reino Unido y USA y encontraron grandes divergencias entre países, tanto en cantidad de la información sobre RSC que se discutía o la importancia que se daba a ser percibidos públicamente como socialmente responsables -mucho más en USA y Reino Unido-, como en las motivaciones expresadas para adoptar una postura responsable y en los asuntos incluidos como prioritarios.

En el caso de los hospitales catalanes, los aspectos observados con más frecuencia fueron el sistema de gestión y el código ético. Cabe también destacar, que casi la mitad de los hospitales de la red pública catalana tienen publicado en la web su plan estratégico. En un estudio editado por la Asociación Española para la Calidad (2011) el porcentaje de empresas que tienen plan estratégico está un poco por encima (69\%).

Respecto a las políticas de transparencia, el 53,3\% de los hospitales publican su rendición de cuentas en la web, aunque no formalizados mediante informes de sostenibilidad, ya que sólo el $28,3 \%$ de los hospitales realizan informes de sostenibilidad. Pero si lo comparamos con los hospitales que utilizan la metodología GRI disminuye al $15 \%$. Estos resultados confirman la necesidad de mayor promoción y formación en la realización de los informes de sostenibilidad. Por otra parte, destacar que la credibilidad de las memorias de RSC depende de la información que presenten, y de si ha sido verificada por un auditor externo o no. En el plano internacional se ha estimado que solo el $40 \%$ de los informes presentados en 2003 incluían evaluaciones externas (Corporate Register, 2004) y en nuestro país ese porcentaje se reducía al $2 \%$ (Fundación Empresa y Sociedad, 2004). En el ámbito de los clientes/usuarios 
la rendición de cuentas es mayor, ya que comprende su participación en encuestas de satisfacción.

En referencia a los aspectos sociales, un aspecto importante es que solo el $18,3 \%$ disponen de plan de igualdad.

En el campo de la acción social, el $26,67 \%$ realiza algún tipo de proyecto solidario y el $3,33 \%$ dispone de un programa de voluntariado corporativo. A nivel general, el número de empresas que realizan acción social se ha mantenido en porcentajes muy altos en la última edición del Estudio Multisectorial sobre el estado de la responsabilidad corporativa de la gran empresa en España publicado por el Club de Excelencia en Sostenibilidad en 2011. En ambos casos, la casi totalidad de las empresas participantes realizan acción social, el $70 \%$ de ellas de forma sistemática y con un plan de acción social. El $91 \%$ de las empresas optan por el formato de la aportación económica para la cofinanciación de proyectos, seguido del $81 \%$ con proyectos propios específicos de proyectos. El $65 \%$ lo hace a través del voluntariado corporativo modalidad ésta que merece una mención por el auge que ha tenido en el último año, apoyado en el Año Europeo (2011) del voluntariado.

La información publicada por las propias instituciones en las webs corporativas, suele ser limitada y poco sistemática, la mayoría de los hospitales más que informar sobre a RSC se limitan a colgar documentos. En este sentido sería conveniente complementar este trabajo en el futuro con estudios sobre la incorporación de prácticas de RSC en la gestión de los hospitales, a través un análisis más profundo de la visión, estrategia y políticas de desempeño de sostenibilidad implementadas por los gestores de los centros sanitarios, mediante entrevistas directas acerca de los contenidos de los informes de sostenibilidad.

Aunque se ha avanzado mucho en la RSC en los centros sanitarios, todavía consideramos que es insuficiente; se requiere unificar criterios como respeto a los contenidos; así como incluir la RSC en la gestión global de los hospitales. Del análisis realizado podemos destacar que los centros sanitarios catalanes se encuentran en una etapa inicial de incorporación e implementación de prácticas de RSC. Se propone un patrón de mínimos sobre RSC que los hospitales tendrían que tener visible en su webs corporativas (tabla 4).

\section{Tabla 4}

Patrón de mínimo de RSC en los hospitales catalanes

\begin{tabular}{cccc}
\hline $\begin{array}{c}\text { Aspectos } \\
\text { generales }\end{array}$ & $\begin{array}{c}\text { Aspectos } \\
\text { económicos }\end{array}$ & Aspectos sociales & $\begin{array}{c}\text { Aspectos } \\
\text { medioambientales }\end{array}$ \\
\hline Política de RSC & $\begin{array}{c}\text { Rendición de } \\
\text { cuentas }\end{array}$ & $\begin{array}{c}\text { Código ético y } \\
\text { código de buenas } \\
\text { prácticas }\end{array}$ & $\begin{array}{c}\text { Política medio } \\
\text { ambiental }\end{array}$ \\
\hline $\begin{array}{c}\text { Gestión de } \\
\text { calidad }\end{array}$ & $\begin{array}{c}\text { Código de buen } \\
\text { gobierno }\end{array}$ & $\begin{array}{c}\text { Conciliación de la } \\
\text { vida familiar y } \\
\text { laboral }\end{array}$ & $\begin{array}{c}\text { Gestión medio } \\
\text { ambiental }\end{array}$ \\
\hline $\begin{array}{c}\text { Memoria de } \\
\text { sostenibilidad }\end{array}$ & $\begin{array}{c}\text { Gestión de la } \\
\text { diversidad }\end{array}$ & \\
\hline $\begin{array}{c}\text { Adhesión del } \\
\text { Pacto Mundial }\end{array}$ & Plan de lgualdad & \\
\hline
\end{tabular}


Responsabilidad social corporativa en los hospitales catalanes: ¿qué nos dicen sus webs?

Plan estratégico

Fuente: Elaboración propia a partir de datos extraídos de las webs corporativas de los hospitales

Distintas iniciativas son las que han servido de referencia en el contexto español, como la Global Reporting Initiative, que con sus guías ha motivado la publicación de los informes de sostenibilidad (143 entidades españolas en el año 2011); el Pacto Mundial de Naciones Unidas, que conlleva la publicación de los informes de progreso (622 entidades españolas en el año 2011), y otros buen número de iniciativas, incluso del ámbito nacional, aplicación del sistema de gestión SGE21, impulsado por Forética (105 entidades españolas en el año 2011), o AENOR, que ha iniciado recientemente la certificación según el referencial IQNet SR $10^{5}$, documento técnico que establece los requisitos para implantar un Sistema de Gestión de la Responsabilidad Social en las organizaciones, apostando por la mejora continua y contemplando de forma sistemática y eficaz la relación con todos los grupos de interés de una organización.

Como se ha señalado a lo largo de este artículo, la RSC es un concepto que, aunque se está extendiendo, es todavía bastante desconocido por parte de los centros sanitarios, lo que provoca que el número de empresas que están desarrollando actuaciones verificables en materia de RSC sea todavía testimonial. Sin embargo, las prácticas de RSC son mucho más habituales, desarrollándose de forma intuitiva, fundamentalmente desde los centros sanitarios, aunque no estén incorporadas dentro de su modelo de gestión. Es un reto poder poner en valor y visualizar todas estas prácticas y las empresas que las desarrollan.

\section{LIMITACIONES}

Como limitación del estudio, cabe mencionar que la información se ha recogido de las webs corporativas de los hospitales en un momento determinado, y que puede haber cambiado. Sabemos que una característica del entorno web es que está en constante evolución y puede que haya habido cambios posteriores que no queden recogidos. Un punto fuerte del estudio es que hemos revisado todas las webs de todos los hospitales públicos catalanes.

\section{CONCLUSIONES}

Aunque se ha avanzado mucho en temas de Responsabilidad social corporativa en los hospitales, observamos que todavía se encuentra poco implantado en los hospitales de Cataluña, sin grandes diferencias en las distintas regiones sanitarias. Además la divulgación de la información sobre prácticas de RSC es muy heterogénea entre los diferentes centros. Se requiere unificar criterios comunes respectos a la RSC, así como acordar una política común sobre la información de RSC en las webs hospitalaria.

La valoración que los grupos de interés hacen de una empresa depende de toda la información que reciben y de las herramientas empleadas para ello. En este sentido, desde nuestro punto de vista, los hospitales deben informar a sus 
grupos de interés de sus prácticas de RSC, ya que además, la rendición de cuenta es una parte esencial de la RSC y las memorias de sostenibilidad son un instrumento fundamental para este fin. En futuras investigaciones se tendría que indagar más en la gestión de la RSC en los hospitales, así como en el dialogo y la comunicación con los grupos de interés.

\section{NOTAS}

1 El triple Botton Line es un término de negocios sustentables que hace referencia al desempeño de una empresa expresado en tres dimensiones: económica, ambiental y social. Su origen en inglés (triple botton line) hace alusión al resultado neto expresado en el último renglón del estado de resultados contables. La expresión fue utilizada por primera vez por John Elkington en 1994, quien posteriormente expandió y articulo a fondo en su libro Cannibal swith forks: The triple bottom line of 21st century business.

2 El Pacto Mundial de Naciones Unidas (Global Compact) es una iniciativa internacional que promueve implementar los 10 principios universalmente aceptados para promover la responsabilidad social empresarial (RSE) en las áreas de Derechos Humanos, Normas laborales, Medio Ambiente y la lucha contra la corrupción en las actividades y la estrategia de negocio de las empresas. Tiene más de 13.000 entidades firmantes con más de 145 países, es la mayor iniciativa voluntaria de responsabilidad social empresarial en el mundo. Esta iniciativa de responsabilidad social empresarial persigue dos objetivos complementarios:1) Incorporar los 10 principios en las actividades empresariales de todo el mundo y 2) Canalizar acciones en apoyo de los objetivos más amplios de las Naciones Unidas, incluidos los objetivos de Desarrollo Sostenible (ODS).

${ }^{3}$ EMAS (Eco-Management and Audit Schema o Reglamento Comunitario de Ecogestión y Ecoauditoría). Es una normativa voluntaria de la Unión Europea que reconoce a aquellas organizaciones que han implantado un sistema de Gestión Medioambiental (SGMA) y han adquirido un compromiso de mejora continua, verificado mediante auditorias independientes.

${ }^{4}$ EFQM (Fundación Europea para la gestión de la Calidad). Fundación sin ánimo de lucro y con sede en Bruselas que cuenta con más de 500 socios repartidos en más de 55 países. Esta fundación define el modelo de Calidad y Excelencia como vía para la autoevaluación y la determinación de los procesos y de mejora continua en entornos empresariales tanto privados como públicos.

${ }^{5}$ IQNet SR 10. Sistema de Gestión de la Responsabilidad Social

\section{REFERENCIAS}

AENOR (2009a). Sistemas de gestión de la Responsabilidad Social de las Empresas Requisitos para el ejercicio de la responsabilidad ética y social. UNE 165010 EX. Madrid: AENOR.

AENOR (2009b). RS 10 Sistema de gestión de la Responsabilidad Social. Requisitos. Madrid: AENOR.

AENOR (2010). Guía sobre la Responsabilidad Social. Especificaciones de Diseño. ISO 26000. Madrid: AENOR. 
Arencibia, M.; Aibar, C. (2007). Páginas Webs de hospitales. Realidad o ¿son realmente un medio útil para la difusión de información para sus usuarios?. Revista de Calidad Asistencial, 22(3), 118-27.

Bhattacharya, C.B. (2003). Does it pay to be a good citizen? A market based view. 2003 International corporate citizenship conference. San Antonio, Texas.

Cencerrado, V. (2002). Las páginas Webs hospitalarias. Web del CHV de Albacete: una apuesta de futuro. Albacete: Complejo Hospitalario Universitario de Albacete. Recuperado de http://www.chospab.es/retransmisiones/index.htm.

Comisión de Trabajo y Asuntos Sociales (2006). Informe de la Subcomisión para potenciar y promover la responsabilidad social de las empresas. Congreso de los Diputados. Serie D, número 424.

Comisión de las Comunidades Europeas (2002). La responsabilidad social de las empresas: una contribución empresarial al desarrollo sostenible. Comunicación de la Comisión de 2 de julio de 2002. Bruselas.

Comité de Industrias Energéticas y el Comité de Gestión Sostenible (2011). Benchmarking RSC Informe final. Madrid: AEC

CSR Europe, Deloitte, Euronext (2003). Investing in responsible business. CSR Europe. Recuperado de http://www.csreurope.org.

Deloitte-ESADE (2012). Análisis sobre la situación actual de las empresas españolas no cotizadas en relación con el Buen Gobierno. Madrid: Deloitte S.L. Recuperado de https://goo.gl/CnpDuc

Elkington, J. (1997). Cannibals with forks: The triple bottom line of 21st century business. Oxford: Capstone Publishing Ltd.

Epstein, E. M. (1987). The Corporate Social Policy Process: Beyond Bussiness Ethics, Corporate Social Responsibility, and Corporate Social Responsiveness. Californian Management Review, 29(3), 99-114.

Esrock, S.L.; Leichty, G. B. (1998). Social Responsibility and Corporate Web Pages: Sejf-Presentation or Agenda-Setting?. Public Relations Review, 24 (3), 305-319

FES (2003). Las empresas mejor percibidas por su acción social. Ranking 2003. Madrid: Fundación Empresa y Sociedad.

FES (2004). La responsabilidad corporativa y la acción social en la Memoria 2003 de las grandes empresas españolas. Madrid: Ed. Fundación Empresa y Sociedad.

Forética (2004). Informe Forética 2004. Responsabilidad social de las empresas. Recuperado de http://www.foretica.org/wpcontent/uploads/2016/01/informe foretica_2004.pdf.

Forética (2011). Informe Forética 2011. Evolución de la responsabilidad social de las empresas en España. Recuperado de http://www.foretica.org/wpcontent/uploads/2016/01/informe_2011_resumen_ejecutivo.pdf.

Foro de Expertos en RSE (2005). Informe del Foro de Expertos en Responsabilidad Social de las Empresas. Madrid: Secretaria General de la Dirección General de Economía Social, Ministerio de Trabajo y Asuntos Sociales. Recuperado de http://www.mtas.es/Empleo/economiasoc/NoticiasDoc/NoticiasPortada/Pri mer_DOC_Foro_Expertos20_7_2005.pdf. 
Responsabilidad social corporativa en los hospitales catalanes: ¿qué nos dicen sus webs?

Global Reporting initiative (2000). Guía para la elaboración de Memorias de Sostenibilidad. Amsterdam: Ed. Global Reporting initiative. Recuperado de https://www.globalreporting.org/resourcelibrary/Spanish-G3.1-Complete.pdf.

Libro Verde (2001). Fomentar un marco europeo para la responsabilidad social de las empresas. Bruselas: Comisión Europea.

Listado actualizado de memoria GRI de organismos públicos. Recuperado de http://database.globalreporting.org/search.

Lozano, J. M.; Albareda, L.; Arenas, D. (2007). Tras la RSE. La responsabilidad social de la empresa en España vista por sus actores. Barcelona: Granica.

Maignan, I.; Ralston, D.A. (2002). Corporate Social Responsibility in Europe and the U.S.: Insigths from Businesses Self-presentations, Journal of International Business Studies, 33, 3-15.

Manheim, J. B.; Pratt, C. B. (1986). Communicating Corporate social Responsibility. Public Relations Review, Summer, 9-18.

Marín Calahorro, F. (2008). Responsabilidad social corporativa y comunicación. Madrid: Fragua.

Moreno, A.; Capriotti, P. (2006). La comunicación de las empresas españolas en sus webs corporativas. Análisis de la información de responsabilidad social, ciudadanía corporativa y desarrollo sostenible. Zer-Revista de Estudios de Comunicación 21, 47-62

PWC (2007). CEO Survey. 6th annual global CEO survey. Leadership, responsibility and growth in uncertain times. New York: Pricewaterhouse Coopers \& World Economics Forum. Recuperado de http://www.pwcglobal.com

United Nations Global Compact (2013). Corporate Sustainibility Report 2013. New York: United Nations Global Compact Office. Recuperado de https://www.unglobalcompact.org/docs/about the gc/Global Corporate S ustainability Report2013.pdf

United Nations Global Compact (2014). United Nations Global Compact Local Network Report 2013. New York: United Nations Global Compact Office. Recuperado de https://www.unglobalcompact.org/docs/publications/LN Report 2013.pdf. 